\title{
Cooperation of IRAKI/4 inhibitor and ABT-737 in nanoparticles for synergistic therapy of $T$ cell acute lymphoblastic leukemia
}

This article was published in the following Dove Press journal:

International Journal of Nanomedicine

31 October 2017

Number of times this article has been viewed

\author{
Xiaoyan $\mathrm{Wu}^{\prime}$ \\ Lin Wang' \\ Yining Qiu' \\ Bingyu Zhang' \\ Zhenhua $\mathrm{Hu}^{2}$ \\ Runming Jin' \\ 'Department of Pediatrics, Union \\ Hospital, Tongji Medical College, \\ Huazhong University of Science and \\ Technology, Wuhan, ${ }^{2}$ Department \\ of Pharmacy, Shanghai Jiao Tong \\ University, Shanghai, China
}

\begin{abstract}
T cell acute lymphoblastic leukemia (T-ALL) is caused by clonal expansion of variant $\mathrm{T}$ cell progenitors and is considered as a high risk leukemia. Contemporary single chemotherapy has a limited effect due to dynamic and versatile properties of T-ALL. Here IRAK1/4 inhibitor and ABT-737 were co-encapsulated into polyethylene glycol modified poly (lactic-co-glycolic acid) nanoparticles (IRAK/ABT-NP) to enhance synergistic therapy of T-ALL. The formulation was optimized to achieve high drug loading using Box-Behnken design and response surface methodology. The optimal parameter comprised $2.98 \%$ polymer in acetonitrile, a ratio of oil phase to water phase of 1:8.33, and 2.12\% emulsifier concentration. High drug loading and uniform spherical shape was achieved. In vitro release study showed sustained release of IRAK1/4 inhibitor for 72 hours as well as sustained release of ABT-737 for more than 120 hours. Uptake efficiency of IRAK/ABT-NP and induced apoptotic T-ALL fraction by IRAK/ABT-NP were much higher than the IRAK1/4 and ABT-737 combined solution. $\mathrm{IC}_{50}$ of IRAK/ABT-NP was twofold lower than free drug combination in Jurkat cells. Additionally, we conducted in vivo experiments in which IRAK/ABT-NP exhibited greater cytotoxicity toward T-ALL cells, the capacity to significantly restore white blood cell number in peripheral blood, and improved survival time of T-ALL mouse model compared to the IRAK1/4 and ABT-737 combined solution.
\end{abstract}

Keywords: T cell acute lymphoblastic leukemia, IRAK1/4 inhibitor, ABT-737, Box-Behnken design and response surface methodology, PEG-PLGA

\section{Introduction}

T cell acute lymphoblastic leukemia (T-ALL) is caused by clonal expansion of variant $\mathrm{T}$ cell progenitors, and is considered as a high risk leukemia which accounts for around $15 \%$ of acute lymphoblastic leukemia cases in children and up to $25 \%$ in adults. ${ }^{1}$ With contemporary chemotherapy, the complete remission rate is as high as $85 \%$ in children, but it is not ideal in adults. ${ }^{2}$ Only $15 \%-25 \%$ of adult T-ALL patients get a good prognosis after relapsing, and the 5-year survival rate is less than $50 \%{ }^{3}$. Therefore, there is an urgent clinical need for more effective therapies for treatment of T-ALL patients.

Currently, there are various kinase inhibitors which have been launched or are in development that target various signaling pathways of cell growth and differentiation. This class of kinase inhibitors is able to impede malignant cell differentiation and proliferation, but is unable to eliminate malignant cells as well as conventional chemotherapy. ${ }^{4}$ However, a combination of kinase inhibitors with chemotherapy drugs has the potential to yield synergistic effects and improve clinical outcome. IRAK4 can activate various transcription factors to promote $\mathrm{T}$ cell survival or proliferation. ${ }^{5}$
Correspondence: Xiaoyan Wu Department of Pediatrics, Union Hospital, Tongji Medical College, Huazhong University of Science and Technology, Wuhan, 430022, China Tel +86 I362868 I72।

Email1994xh0558@hust.edu.cn 
IRAK1/4 inhibition has been shown to significantly impair malignant $\mathrm{T}$ cell line proliferation, but has no significant effects on cell viability of malignant T cells in vitro. ${ }^{6-9}$ When combining IRAK1/4 inhibitor with therapeutic compounds, the cytotoxic activity of various types of antileukemic drugs was augmented by IRAK1/4 inhibitor. ABT-737, an agent that disrupts microtubules, has the best synergistic effect with IRAK1/4 inhibitor in killing T-ALL cells due to the combination of BCL2 and BCL-xL impairment by ABT737 and the dramatic decrease of MCL1 levels by IRAK1/4 inhibitor. ${ }^{7,10-12}$

Despite the improvement in cytotoxic effect on malignant T-ALL cells by the combination of the IRAK $1 / 4$ inhibitor and ABT-737, a nanoparticle based drug delivery system has been proven to increase the effect of chemotherapy drugs through enhancing permeability and retention in tumor cells, improving pharmacokinetic profiles, and reducing side effects. ${ }^{13-18}$ Therefore, here we co-encapsulated IRAK1/4 inhibitor and ABT-737 into biodegradable and biocompatible polyethylene glycol (PEG) modified poly (lactic-co-glycolic acid) (PEGPLGA) polymer nanoparticles (IRAK/ABT-NP) as a novel and advanced therapy strategy for T-ALL treatment. The efficacy of IRAK/ABT-NP was assessed in a T-ALL cell line xenograft mouse model.

\section{Materials and methods}

\section{Materials}

IRAK-1/4 Inhibitor I was purchased from Sigma-Aldrich Co. (St Louis, MO, USA). ABT-737 was purchased from Abcam (Shanghai, China). RPMI 1640 and FBS were obtained from Thermo Fisher Scientific (Waltham, MA, USA). FITC-AnnexinV and 7-AAD were ordered from Invitrogen (Thermo Fisher Scientific). PEG-PLGA was purchased from PolySciTech (West Lafayette, IN, USA). Other chemical reagents were purchased from Sigma-Aldrich Co.

\section{Preparation of IRAK/ABT-NP}

IRAK/ABT-NP containing IRAK1/4 inhibitor and ABT-737 were prepared by emulsion-solvent evaporation method. ${ }^{19}$ In brief, PLGA polymer, IRAK1/4 inhibitor, and ABT-737 were dissolved in $3 \mathrm{~mL}$ of acetonitrile, and emulsified in $12 \mathrm{~mL}$ of $2 \%(\mathrm{w} / \mathrm{v})$ poly(vinyl alcohol) solution. Emulsification was performed using a micro-tip probe sonicator (VC505; Vibracell Sonics, Newtown, CT, USA) with $70 \mathrm{~W}$ of energy output for 3 min over an ice bath. The emulsion was stirred for 16 hours at room temperature to evaporate organic solvent and get solid nanoparticles. Nanoparticles were collected by ultracentrifugation at $30,000 \mathrm{rpm}$ for $30 \mathrm{~min}$ at $4^{\circ} \mathrm{C}$ (Sorvall Ultraspeed Centrifuge; Kendro, Weaverville,
NC, USA) and washed twice with distilled water followed by lyophilization for storage.

\section{Experimental design}

Box-Behnken design and response surface methodology (BBDRSM) was used to optimize IRAK/ABT-NP. ${ }^{20}$ According to reviewed studies, the following variables were selected: polymer concentration, ratio of oil phase to water phase, and emulsifier concentration. For each variable, three levels were chosen for Box-Behnken experiment design, as shown in Table 1. Drug loading was measured as response values (Y). Minitab 18 software was applied for experimental design. The effects of independent variables on the responses were evaluated using analysis of variance (ANOVA), with statistical significance established at $p<0.05 .{ }^{21}$ The fitness of model was evaluated by calculating predicted and adjusted correlation coefficient $\left(R^{2}\right)$. To display the experimental region and effects of independent variables on the response, three-dimensional response surface graphs and contour plots were drawn. To verify the optimal formulation, the experiment was conducted in triplicate responses to evaluate accuracy of the predicted value.

\section{Scanning electron microscopy (SEM)}

Morphology of IRAK/ABT-NP was analyzed by SEM. IRAK/ABT-NP were suspended in deionized water. A drop of the suspension was put on a glass slide until it dried at room temperature. Then, the dried samples were sputtered with gold and placed on a copper stub. SEM images were acquired at $5 \mathrm{kV}$ sputtering energy under high vacuum.

\section{Transmission electron microscopy (TEM)}

The internal structure of IRAK/ABT-NP was characterized by TEM. IRAK/ABT-NP were suspended with deionized water. A drop of the formulation was placed on a carbon coated copper grid. Then, the samples were negatively stained with $2 \%$ phosphotungstic acid. After drying, TEM images were acquired at a voltage of $120 \mathrm{kV}$ under a microscope.

\section{Assessing drug loading of IRAKI/4 inhibitor and ABT-737}

Reverse phase-High-performance liquid chromatography (RP-HPLC) was used to estimate the amount of encapsulated

Table I Selective variables level in Box-Behnken design

\begin{tabular}{lllll}
\hline Variables & Factors & \multicolumn{3}{l}{ Code } \\
\cline { 3 - 5 } & & $-\mathbf{I}$ & $\mathbf{0}$ & $\mathbf{I}$ \\
\hline A & Polymer concentration (\%) & 0.5 & 2 & 3.5 \\
B & Oil phase:water phase (V/V) & I:I & I:7 & I:I3 \\
C & Emulsifier concentration (\%) & 0.5 & 2 & 3.5 \\
\hline
\end{tabular}


IRAK1/4 inhibitor and ABT-737 in IRAK/ABT-NP. An amount of $2 \mathrm{mg}$ of lyophilized IRAK/ABT-NP was dissolved in $1 \mathrm{~mL}$ of DMSO solution. ${ }^{22}$ The sample was sonicated for $2 \mathrm{~min}$ and then centrifuged for $10 \mathrm{~min}$ at $10,000 \mathrm{rpm}$ and the supernatant was analyzed by RP-HPLC. A mobile phase including $0.1 \% \mathrm{H}_{3} \mathrm{PO} 4$ in acetonitrile and $0.1 \% \mathrm{H}_{3} \mathrm{PO} 4$ in water was utilized..$^{22}$ The ratio of acetonitrile and water was 90:10 (v/v). A quaternary pump was used to deliver the mobile phase at a flow rate of $0.8 \mathrm{~mL} / \mathrm{min}$ at $25^{\circ} \mathrm{C}$. ABT-737 levels were measured by UV detection at $300 \mathrm{~nm}$. IRAK1/4 inhibitor levels were measured by UV detection at $254 \mathrm{~nm}$. A standard curve of IRAK1/4 inhibitor and ABT-737 also needed to be established under the same conditions. The amount of IRAK1/4 inhibitor and ABT-737 in nanoparticles can be estimated by correlating the peak area with the standard curve. The result was divided by the total amount of drug supplied in the formulation and then multiplied by 100 to calculate the drug loading of IRAK1/4 inhibitor and ABT-737. All analyses were performed in triplicate.

\section{Particle size analysis and zeta potential measurement}

The hydrodynamic diameter was measured with dynamic light scattering (DLS), and zeta potential (mV) was determined by laser Doppler anemometry (LDA). A Malvern Zetasizer was used to perform the DLS and LDA analyses. IRAK/ABT-NP were diluted with double distilled water into a suspension to reach a concentration of $50 \mathrm{ng} / \mathrm{mL}$ and sonicated for $30 \mathrm{~s}$ in an ice bath. Particle size and zeta potential were measured with the Malvern Zetasizer. All measurements were performed in triplicate.

\section{In vitro release study}

In vitro release of either IRAK1/4 inhibitor or ABT737 from optimized IRAK/ABT-NP was carried out with a dialysis method. Before the experiment, dialysis membranes (molecular weight cut-off $10 \mathrm{kDa}$ ) were immersed in distilled water overnight at room temperature and rinsed thoroughly to remove the preservative. After IRAK/ABT-NP were put inside, the dialysis bag was sealed tightly. Then, it was immersed in a medium consisting of $50 \mathrm{~mL}$ of PBS (0.01 M, pH 7.4) containing $0.1 \% \mathrm{v} / \mathrm{v}$ of Tween 80 (to ensure a sink condition) and kept in a shaking incubator at $37^{\circ} \mathrm{C}$ and $100 \mathrm{rpm} .{ }^{23} \mathrm{At}$ scheduled time intervals, $0.5 \mathrm{~mL}$ of medium was taken out from the shaking incubator, and the same volume of fresh medium was added immediately. The samples were dissolved in $1 \mathrm{~mL}$ of acetonitrile solution. The content of
IRAK1/4 inhibitor or ABT-737 in the diluted sample was analyzed by RP-HPLC method, described in the "Assessing drug loading of IRAK1/4 inhibitor and ABT-737" section. The experiment was performed in triplicate.

\section{Cell culture}

Human T cell leukemia cell line (Jurkat) was provided by (Yanyu Biology Technology Co., Ltd, Shanghai, China) and cultured in RMPI 1640 media supplemented with 10\% FBS, and $1 \%$ penicillin-streptomycin, in an incubator at $37^{\circ} \mathrm{C}$, in a humidified, $5 \% \mathrm{CO}_{2}$ atmosphere. Chemicals for cell culture were purchased from Sigma-Aldrich Co.

\section{Cellular uptake by fluorescence spectrophotometry}

Fluorescently labeled IRAK1/4 inhibitor was used to measure uptake of IRAK/ABT-NP in Jurkat cells. Jurkat cells were seeded in a plate with four chambers and incubated at $37^{\circ} \mathrm{C} .{ }^{24}$ The cells were treated, respectively, with IRAK1/4 inhibitor and ABT-737 combined solution, or IRAK/ABT-NP with the same concentration of drugs. The cells were incubated for 4 hours and then washed three times with PBS to remove drugs and particles. Next, the cells were stained with $\mathrm{N}$, $\mathrm{N}$-Diisopropylethylamine for $5 \mathrm{~min}$ and washed three times. A fluorescence microscope was used to observe the uptake of IRAK/ABT-NP in Jurkat cells.

\section{Cytotoxicity study}

The cytotoxicity studies were analyzed by MTT based colorimetric assay. Different concentrations of IRAK1/4 inhibitor and ABT-737 in solution and in IRAK/ABT-NP were tested. ${ }^{25}$ Jurkat cells were seeded in 96-well plates and incubated for 24 hours. The cells were treated with each concentration of drugs and incubated for 48 hours. PBS treated cells were set as controls. The medium was removed carefully after centrifugation and supplemented with fresh medium $(100 \mu \mathrm{L})$. Then, $10 \mu \mathrm{L}$ of $5 \mathrm{mg} / \mathrm{mL}$ MTT was added to each well and incubated for 3 hours at $37^{\circ} \mathrm{C}$. The medium was removed after centrifuging again and $100 \mu \mathrm{L}$ of DMSO was added to each well. A 96-well format ELISA plate reader was used to measure the absorbance at $590 \mathrm{~nm}$ with a reference filter of $620 \mathrm{~nm}$. The results were analyzed by comparing the percentage of metabolically active cells in the treated groups with control cells.

\section{Apoptosis analysis by flow cytometry}

Apoptosis was analyzed with Annexin V-FITC/7-AAD double staining. Briefly, Jurkat cells $\left(1 \times 10^{5}\right.$ cells $\left./ \mathrm{mL}\right)$ were seeded in 6-well plates and treated with PBS, IRAK1/4 
inhibitor, ABT-737, combined treatment, and IRAK/ ABT-NP with an equivalent concentration of ABT-737 for 24 hours. Then, cells were stained with Annexin V-FITC/7AAD dye according to manufacturer's instructions. Cells treated with PBS were set as the control. Finally, cells were harvested and run through the flow cytometer. ${ }^{26}$

\section{Mice}

Jurkat cells $\left(3 \times 10^{6}\right)$ were injected intravenously into 40 female NPG mice (Beijing Vitalstar Biotechnology, Beijing, China) to generate human cell line xenograft T-ALL mouse models. ${ }^{27-29}$ After 7 days, the mice were treated with IRAK inhibitor $(10 \mathrm{mg} / \mathrm{kg})$, ABT-737 (40 mg/kg), IRAK inhibitor and ABT-737 combined solution (10 mg/kg IRAK inhibitor and $40 \mathrm{mg} / \mathrm{kg}$ ABT-737), IRAK/ABT-NP (10 mg/kg IRAK inhibitor and $40 \mathrm{mg} / \mathrm{kg}$ ABT-737), or PBS as control, two times every week. ${ }^{5}$ After 4 weeks, peripheral blood samples from each treated group of mice were taken for counting white blood cells (WBC) and staining in accordance with the Wright-Giemsa method. All animal studies described were in accordance with guidelines determined by the Animal Welfare Act and the Guide for the Care and Use of Laboratory Animals, and approved by the Committee on the Ethics of Animal Experiments of Tongji Medical College, Huazhong University of Science and Technology.

\section{Statistics}

Statistical analysis was performed using the SPSS software (11.5). Statistical significance $(p<0.05)$ before and after treatment was evaluated using the paired Student's $t$-test. The differences between the groups were assessed with ANOVA.

\section{Results}

\section{Optimization of formulation for IRAK/ABT-NP}

IRAK/ABT-NP were prepared from PEG-PLGA polymer using an emulsion-evaporation method. To reduce the injection amount of polymer, IRAK/ABT-NP should have drug loading as high as possible. The response surface methodology was performed to obtain the optimal formulation for IRAK/ABT-NP. The Box-Behnken design form and response values (Y) of drug loading from a total of 15 experiments are presented in Table 2. Analysis of the data of Box-Behnken design was performed using Minitab 18 software. The following equation in coded units was generated for predicting maximum of $\mathrm{Y}$.
Table 2 Box-Behnken design and matrix and corresponding responses

\begin{tabular}{lllll}
\hline Run & \multicolumn{2}{l}{ Variables } & & Responses \\
\cline { 2 - 4 } & A & B & C & Y (\%) \\
\hline I & 0 & 0 & 0 & 7.5 \\
2 & -1 & 1 & 0 & 2.7 \\
3 & -1 & -1 & 0 & 0.9 \\
4 & 1 & 0 & -1 & 5.4 \\
5 & -1 & 0 & 1 & 1.5 \\
6 & 0 & 1 & -1 & 5.5 \\
7 & 0 & -1 & -1 & 5.3 \\
8 & 1 & 1 & 0 & 5.6 \\
9 & 1 & 0 & 1 & 6.4 \\
10 & 1 & -1 & 0 & 5.7 \\
11 & 0 & 1 & 1 & 6.5 \\
12 & 0 & 0 & 0 & 7.4 \\
13 & 0 & -1 & 1 & 5.3 \\
14 & -1 & 0 & -1 & 1.4 \\
15 & 0 & 0 & 0 & 7.3 \\
\hline
\end{tabular}

Notes: $Y$ is drug loading (\%), and A, B and $C$ are coded values for polymer concentration (\%), oil phase:water phase (V/V), and emulsifier concentration (\%), respectively.

$\mathrm{Y}$ is drug loading (\%), and $\mathrm{A}, \mathrm{B}$ and $\mathrm{C}$ are coded values for polymer concentration (\%), oil phase:water phase (V/V), and emulsifier concentration (\%), respectively.

ANOVA was tested using response surface regression, and the results are presented in Table 3 . The model equation has an $F$-value of 183.07 and a $p$-value of 0 , which indicates that it was statistically significant. Lack-of-fit value of the model has a $p$-value of 0.138 exceeding the confidence level of 0.05 . This implies that the model equation was well fitted to predict the maximum of $\mathrm{Y}$ without significant lack items.

Table 3 ANOVA results for response $Y$ (drug loading)

\begin{tabular}{llllll}
\hline Source & df & Adj SS & Adj MS & F-value & p-value \\
\hline Model & 9 & 70.0235 & 7.7804 & 183.07 & 0 \\
Linear & 3 & 36.1975 & 12.0658 & 283.9 & 0 \\
A & $\mathrm{I}$ & 34.445 & 34.445 & 810.47 & 0 \\
B & $\mathrm{I}$ & 1.2013 & 1.2013 & 28.26 & 0.003 \\
C & $\mathrm{I}$ & 0.5513 & 0.5513 & 12.97 & 0.016 \\
Square & 3 & 32.471 & 10.8237 & 254.67 & 0 \\
A*A & $\mathrm{I}$ & 29.4669 & 29.4669 & 693.34 & 0 \\
B*B & $\mathrm{I}$ & 2.6677 & 2.6677 & 62.77 & 0.001 \\
C*C & $\mathrm{I}$ & 2.9908 & 2.9908 & 70.37 & 0 \\
2-way interaction & 3 & 1.355 & 0.4517 & 10.63 & 0.013 \\
A*B & $\mathrm{I}$ & 0.9025 & 0.9025 & 21.24 & 0.006 \\
A*C & $\mathrm{I}$ & 0.2025 & 0.2025 & 4.76 & $0.08 \mathrm{I}$ \\
B*C & $\mathrm{I}$ & 0.25 & 0.25 & 5.88 & 0.06 \\
Error & 5 & 0.2125 & 0.0425 & & \\
Lack-of-fit & 3 & 0.1925 & 0.0642 & 6.42 & 0.138 \\
Pure error & 2 & 0.02 & 0.01 & & \\
Total & $\mathrm{I} 4$ & 70.236 & & & \\
\hline Notes & & & &
\end{tabular}

Notes: $R^{2}=0.9970$, adjusted $R^{2}=0.9915$, and predicted $R^{2}=0.9555$. $Y$ is drug loading (\%), and $A, B$ and $C$ are coded values for polymer concentration (\%), oil phase:water phase (V/V), and emulsifier concentration (\%), respectively.

Abbreviations: $d f$, degrees of freedom; Adj SS, adjusted sum of squares; Adj MS, adjusted mean square. 
According to the coefficient $R^{2}$ value of $0.9970,99.70 \%$ of variability in drug loading of IRAK/ABT-NP can be explained by this model equation. The adjusted $R^{2}=0.9915$ was close in value to the predicted $R^{2}=0.9555$, which ensured high correlation between observed and predicted values in this model, since the difference is below $0.2 .{ }^{30}$ The interaction of $A$ and $B$ factors has a $p$-value of 0.006 which suggests that $A$ and $B$ factors had cross-impact on response value.

To further analyze how each independent variable affects response value, three-dimensional response surface plots and contour plots were graphed using Minitab 18 software to display the relationship between three variables and response value Y. As shown in the three-dimensional response surface plots (Figure $1 \mathrm{~A}-\mathrm{C}$ ), the biggest change range in $\mathrm{Y}$ value is caused by variable $\mathrm{A}$, which indicates that polymer concentration is the main factor affecting drug loading of IRAK/ABT-NP. Variable B has the second largest effect on response value $\mathrm{Y}^{31}$ Comparing areas of $\mathrm{Y}$ value more than 7.5 in contour plots for $\mathrm{Y}$, variable $\mathrm{B}$ and $\mathrm{C}$ have a small variable range at a fixed level of variable A (Figure 1D-F). It was also confirmed that variable $\mathrm{A}$ is the main factor. Then, we calculated the coded variables for the optimized formulation through solving the model equations and found that $\mathrm{A}=0.65, \mathrm{~B}=0.19$, and $\mathrm{C}=0.82$. Optimized IRAK/ABT-NP parameters comprised of $2.98 \%$ polymer in acetonitrile, 1:8.33 oil phase to water phase ratio, and $2.12 \%$ emulsifier concentration based on the prediction model. Experiments were performed in triplicate according to the optimized formulation to evaluate the prediction accuracy of the model equation. Predicted and experimental response values are presented in Table 4. Observed experimental values were in close agreement with the predicted values (relative standard deviation $<2 \%$ ). Agreement between the results indicate the significance and validity of the model.
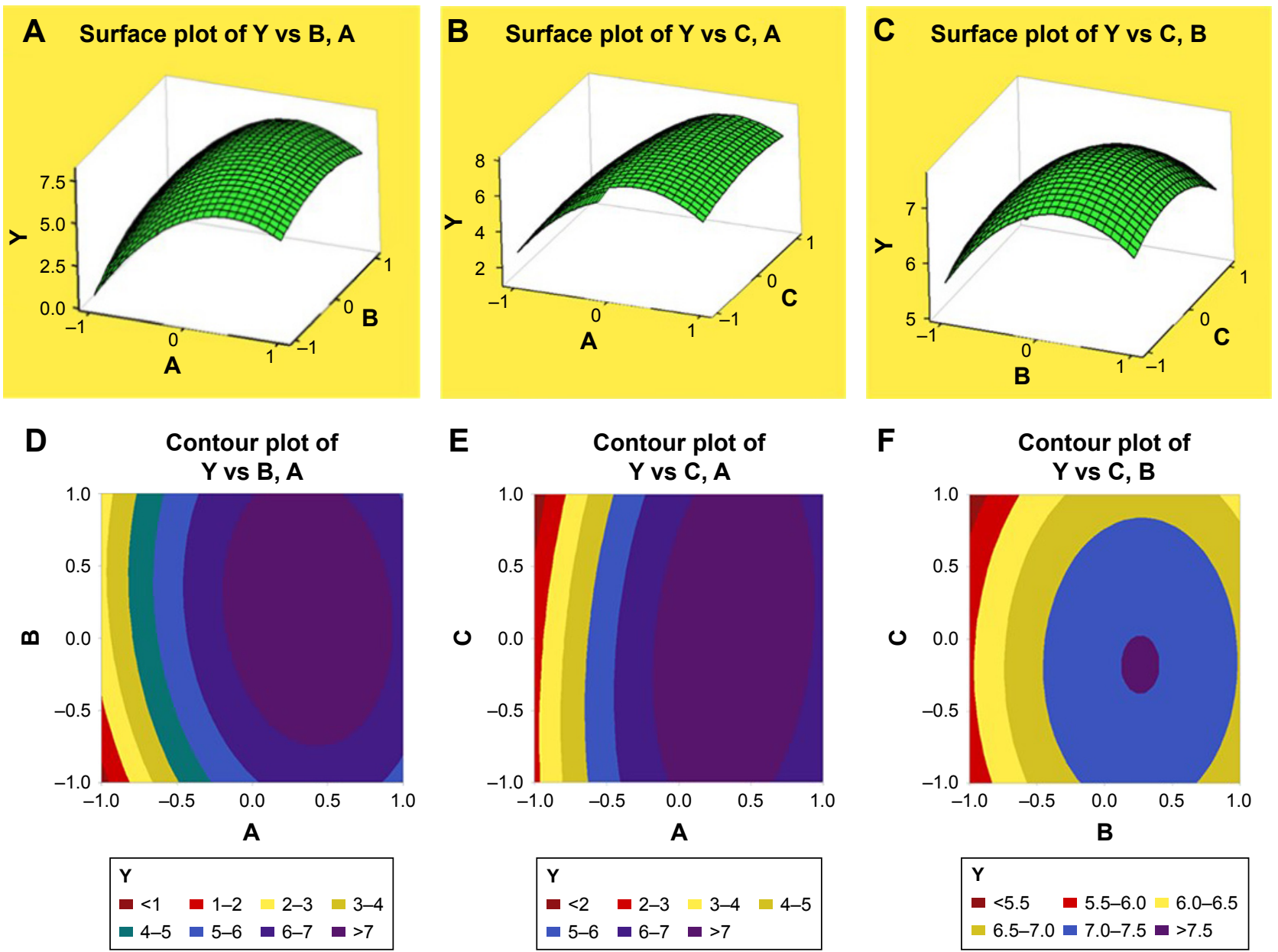

Figure I Three-dimensional response surface plots and contour plots for $Y$ as a function of two of three variables at a fixed value of the third one variable.

Notes: Response surface plot for $Y$ as a function of (A) polymer concentration and volume ratios of oil phase to water phase at fixed emulsifier concentration, (B) polymer concentration and emulsifier concentration at fixed volume ratios of oil phase to water phase, (C) volume ratios of oil phase to water phase and emulsifier concentration at fixed polymer concentration, (D-F) contour plots for $\mathrm{Y}$ as a function of two of three variables at a fixed value of the third one variable as (A-C). $Y$ is drug loading (\%), and $\mathrm{A}, \mathrm{B}$ and $\mathrm{C}$ are coded values for polymer concentration (\%), oil phase:water phase (V/V), and emulsifier concentration (\%), respectively. 
Table 4 Predicted and experimental response values of optimized condition

\begin{tabular}{llll}
\hline Response & $\begin{array}{l}\text { Predicted } \\
\text { value }\end{array}$ & $\begin{array}{l}\text { Experimental } \\
\text { value }\end{array}$ & $\begin{array}{l}\text { Prediction } \\
\text { error (\%) }\end{array}$ \\
\hline$Y$ & 7.31 & 7.4 & 0.12 \\
\hline
\end{tabular}

Notes: Prediction error, relative standard deviation $<2 \%$. $\mathrm{Y}$ is drug loading (\%).

\section{Characterization of IRAK/ABT-NP}

DLS analysis revealed that the optimal IRAK/ABT-NP formulation had an average diameter of $100 \mathrm{~nm}$ and a zeta potential of $-5.1 \mathrm{mV}$. Additionally, surface morphology was studied by SEM (Figure 2A) and TEM (Figure 2C), revealing that particles were spherical with a smooth surface and monodispersed, which was consistent with the result of DLS analysis (Figure 2B). The confocal images of IRAK1/4 inhibitor and ABT-737 labeled with different fluorescent dyes showed the status of these two drugs in PEG-PLGA polymer nanoparticles (Figure 2D). Both IRAK1/4 inhibitor and ABT737 showed sustained release kinetics from PEG-PLGA NP (Figure 2E). Approximately $35 \%$ and $25 \%$ of IRAK1/4 inhibitor and ABT-737 were released from IRAK/ABT-NP respectively within 24 hours. After 24 hours, IRAK/ABT-NP presented slower sustained release profiles. Over $80 \%$ of IRAK1/4 inhibitor was released after 72 hours, but less than $60 \%$ of ABT- 737 was after 120 hours.

\section{Cellular uptake of IRAK/ABT-NP in Jurkat cells}

Fluorescently labeled ABT- 737 was used to evaluate the uptake of combined IRAK1/4 inhibitor and ABT-737 solution and IRAK/ABT-NP in Jurkat cells. ABT-737 in IRAK/ABT-NP showed more cellular uptake than ABT-737 in the combined solution (Figure 3A). Cells treated with IRAK/ABT-NP had approximately nine times higher cellular uptake of ABT-737 than cells treated with combined solution (Figure 3B).

\section{Cell growth inhibition of IRAK/ABT-NP}

Cell growth inhibition following IRAK/ABT-NP treatment was evaluated in Jurkat cells by MTT analysis method and was compared to cell growth inhibition following treatment with IRAK1/4 inhibitor alone or IRAK1/4 and ABT-737 combined solution (Figure 4). The IRAK1/4 inhibitor alone had almost no killing efficacy in Jurkat cells. $\mathrm{IC}_{50}$ of the combined solution was $3.8 \mu \mathrm{M}$, which is much less than the $5.7 \mu \mathrm{M} \mathrm{IC}_{50}$ of ABT-737 alone. These results indicate that IRAK1/4 inhibitor augments the cytotoxic activity of ABT737 in the combined solution. However, IRAK/ABT-NP had the lowest $\mathrm{IC}_{50}$ of $1.7 \mu \mathrm{M}$, which suggests that it has the most potential killing ability of Jurkat cells compared to the other formulations.
A

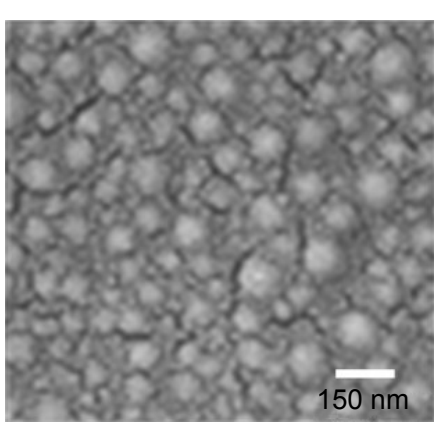

B

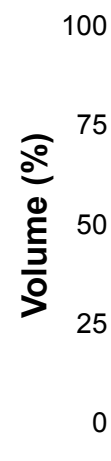

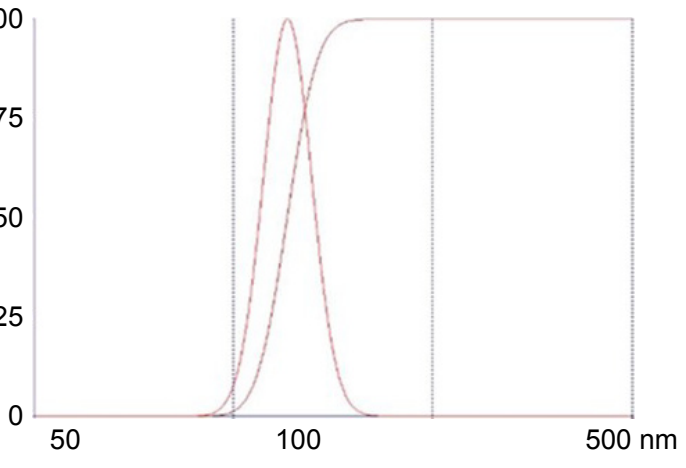

E

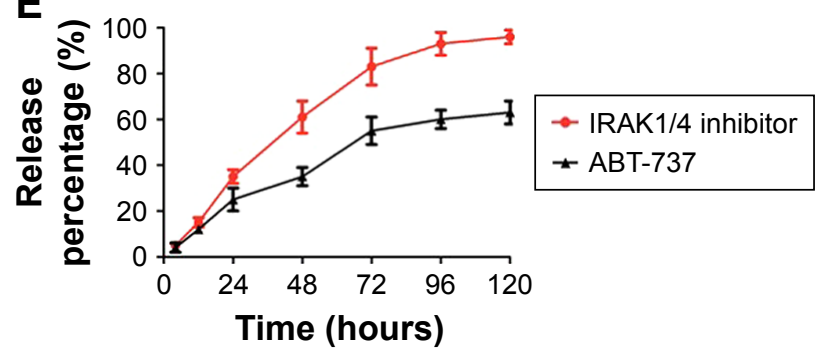

Figure 2 Characterization of IRAK/ABT-NP

Notes: (A) SEM image of IRAK/ABT-NP, (B) size and size distribution of IRAK/ABT-NP, (C) TEM image of IRAK/ABT-NP. (D) Confocal laser scanning microcopy images of fluorescently labeled IRAKI/4 inhibitor and ABT-737 co-encapsulated in IRAK/ABT-NP, (E) In vitro release of drugs from IRAK/ABT-NP.

Abbreviations: IRAK/ABT-NP, IRAKI/4 inhibitor and ABT-737 co-encapsulated into polyethylene glycol modified poly (lactic-co-glycolic acid) nanoparticles; SEM, scanning electron microscopy; TEM, transmission electron microscopy. 

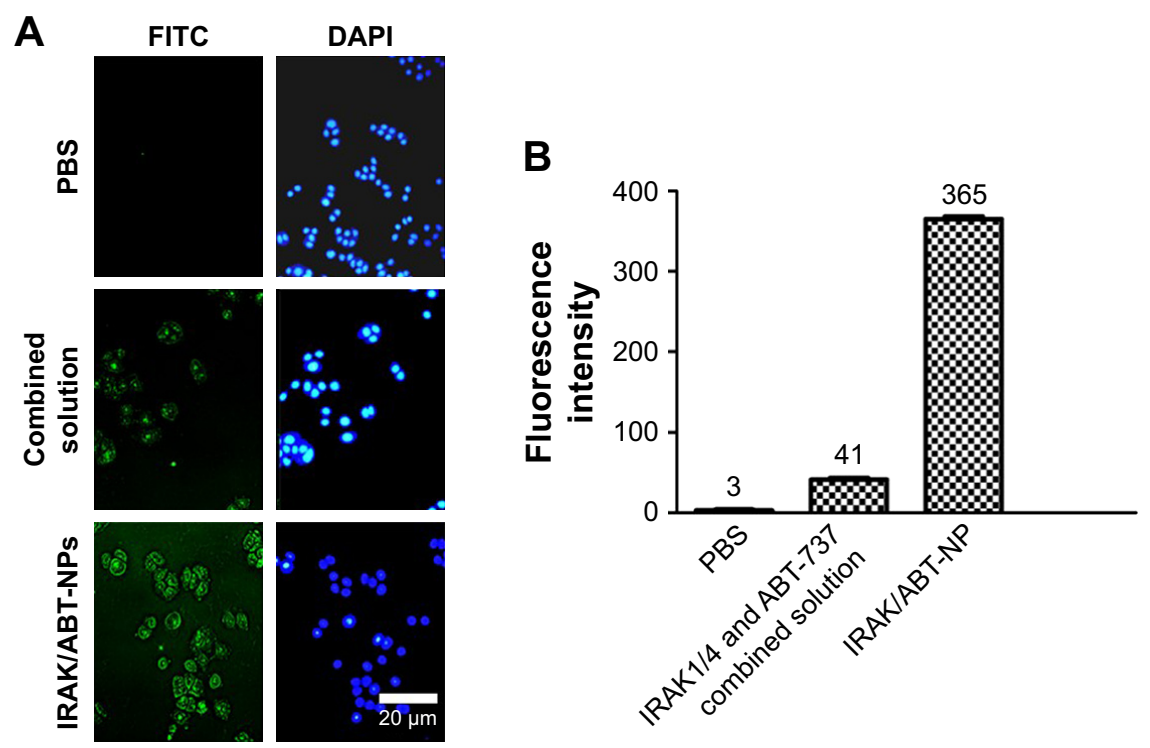

Figure 3 (A) Representative fluorescence images of uptake of IRAK/ABT-NP in Jurkat cells compared to the IRAKI/4 and ABT-737 combined solution, (B) fluorescence intensity analysis of images from $(\mathbf{A})$.

Abbreviation: IRAK/ABT-NP, IRAKI/4 inhibitor and ABT-737 co-encapsulated into polyethylene glycol modified poly (lactic-co-glycolic acid) nanoparticles.

\section{Cell apoptosis induced by IRAK/ABT-NP} Annexin V-FITC and 7-AAD double staining was performed to determine the induced apoptosis in Jurkat cells following treatment with PBS, IRAK1/4 inhibitor, ABT-737, combined solution, and IRAK/ABT-NP. Flow cytometry was used to analyze the percentage of apoptosis (Figure 5). In the PBS treated cells group, the percentage of double negative (Annexin $\mathrm{V}$ and 7-AAD cells negative) live cells was as high as $99.6 \%$. After treatment with IRAK1/4 inhibitor,

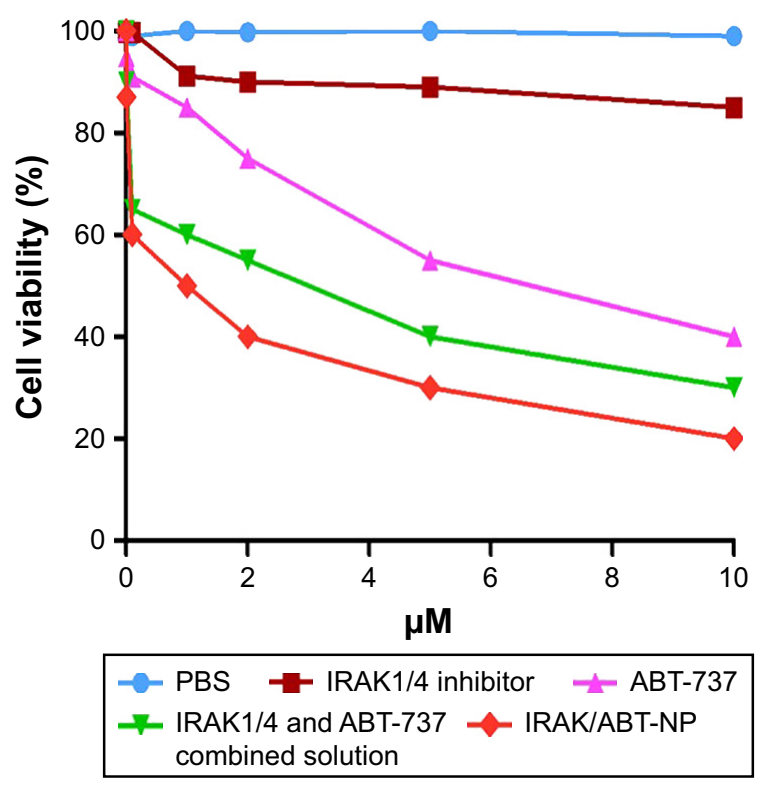

Figure $\mathbf{4}$ In vitro cell viability of Jurkat cells treated with IRAK/ABT-NP, IRAKI/4 inhibitor alone, $A B T-737$ alone and combined solution at different concentrations. Abbreviation: IRAK/ABT-NP, IRAKI/4 inhibitor and ABT-737 co-encapsulated into polyethylene glycol modified poly (lactic-co-glycolic acid) nanoparticles. the percentage of Annexin $\mathrm{V}$ positive live cells increased to $90 \%$, but there were no 7-AAD positive cells. This suggests that IRAK1/4 inhibitor could not induce late apoptosis in Jurkat cells. In the group of cells treated with ABT-373, 7 -AAD positive cells increased to $6.8 \%$, while in the cells treated with combined solution apoptotic cells increased to $39 \%$. However, IRAK/ABT-NP increased apoptotic cells up to $86.4 \%$. These data show that IRAK/ABT-NP induced the greatest proportion of late apoptotic cells in Jurkat cells.

\section{Efficacy in PDX mouse model}

To evaluate efficacy of IRAK/ABT-NP in Jurkat cell xenograft mouse models, WBC in peripheral blood were measured after 4 weeks of treatment. WBC number in mice treated with IRAK1/4 inhibitor alone and ABT-737 alone showed no significant difference compared to PBS treated mice (Figure 6B). WBC number was significantly decreased in the ombined solution treated mice compared to PBS treated mice. Approximately $30 \%$ of mice showed a recovery of WBC number to the normal range of $2.6-10 \times 10^{3} / \mu \mathrm{L}$ after treatment with IRAK/ABT-NP. ${ }^{32}$ The WBC data suggest that IRAK/ABT-NP could better improve lymphoid blast in T-ALL mouse model than other formulations. To confirm the improved efficacy of IRAK/ABT-NP in the treatment of T-ALL mouse model, smears of peripheral blood from each group were prepared to check preliminary impression of numbers of lymphoblastic leukemia cells after 4 weeks' treatment. The blue color cells indicate lymphoblastic leukemia cells stained by Wright-Giemsa method (Figure 6A). 

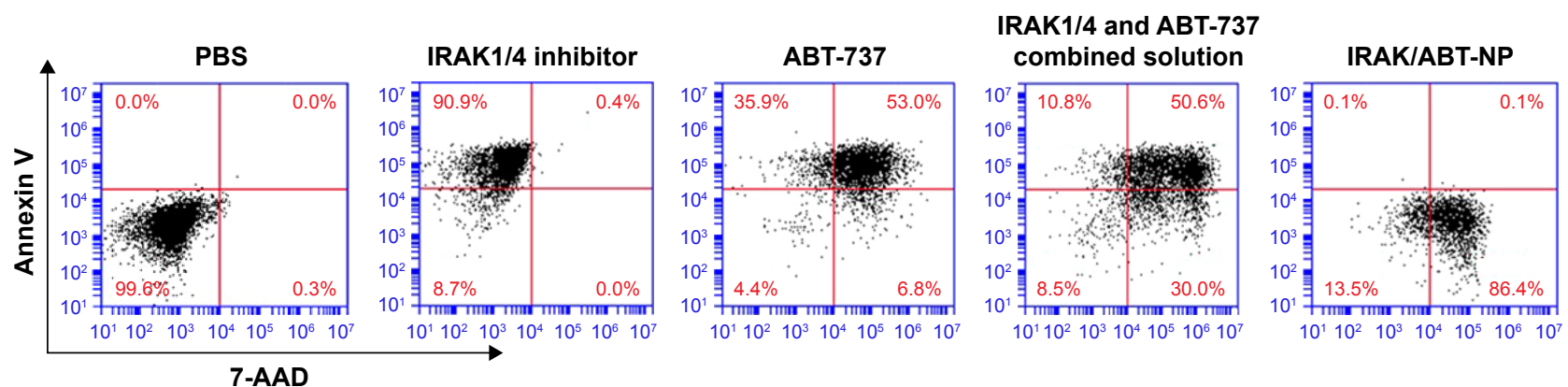

Figure 5 Annexin V/7-AAD staining used for flow cytometry analysis of apoptosis in Jurkat cells induced by IRAK/ABT-NP compared to IRAKI/4 inhibitor alone, ABT-737 alone and combined solution.

Abbreviation: IRAK/ABT-NP, IRAKI/4 inhibitor and ABT-737 co-encapsulated into polyethylene glycol modified poly (lactic-co-glycolic acid) nanoparticles.

Therefore, it can be seen that combined solution treatment reduced amounts of lymphoblastic leukemia cells. But the amounts had almost no change in mice treated with IRAK1/4 inhibitor alone and ABT-737 alone, which was consistent with WBC results. Most of the lymphoblastic leukemia cells disappeared in IRAK/ABT-NP treated mice. The mice with IRAK/ABT-NP treatment showed significantly longer median survival compared to the mice treated with combined solution (50 days vs 65 days, $p<0.0001$ by Log-rank test) (Figure 6C). All these data support IRAK/ABT-NP as having the best potential therapeutic efficacy in T-ALL mouse model.

\section{Discussion}

Combination therapies have become increasingly popular in the treatment of cancer due to the limitations of single drugs in treating the dynamic and versatile properties of malignant tumors. ${ }^{33}$ ABT-737 can induce cell apoptosis through selective inhibition of Bcl-xL and BCL-2, while also having no effect on MCL1. IRAK1/4 inhibitor can dramatically reduce MCL1 level in T-ALL cells. ${ }^{34,35}$ Therefore, combining these drugs can generate a synergetic effect on killing T-ALL cells. Using this knowledge, IRAK $1 / 4$ inhibitor and ABT-737 were co-encapsulated into PEG-PLGA polymer nanoparticles to further enhance the synergetic effect on killing T-ALL cells and improve patient complication. IRAK1/4 inhibitor and ABT-737 are both poorly soluble in water, which will facilitate high encapsulation efficiency in the polymer nanoparticles. ${ }^{36}$ This is the reason why only drug loading was selected as a response value when we used BBD-RSM to optimize the formulation of IRAK/ABT-NP. The release

A

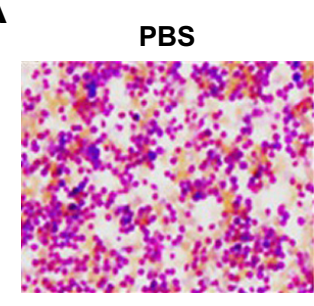

B

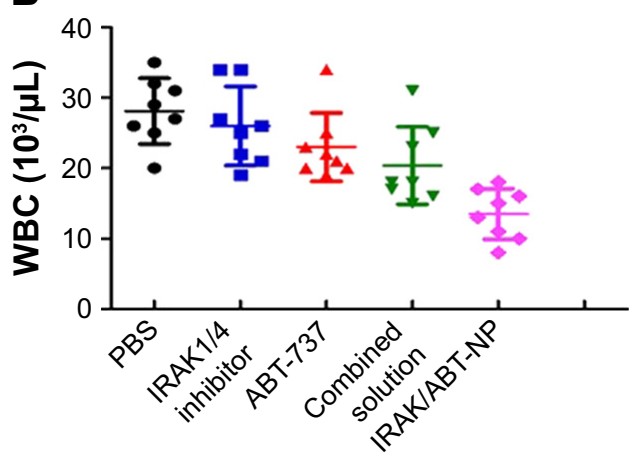

IRAK1/4 inhibitor
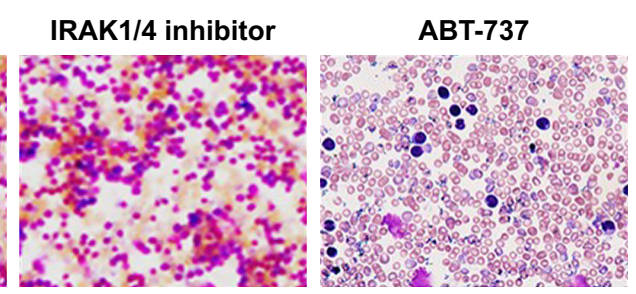

IRAK1/4 and ABT-737 combined solution IRAKIABT-NP

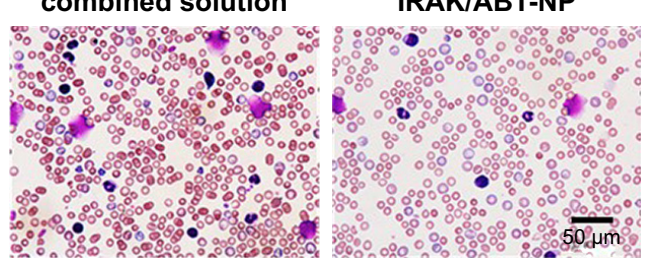

C

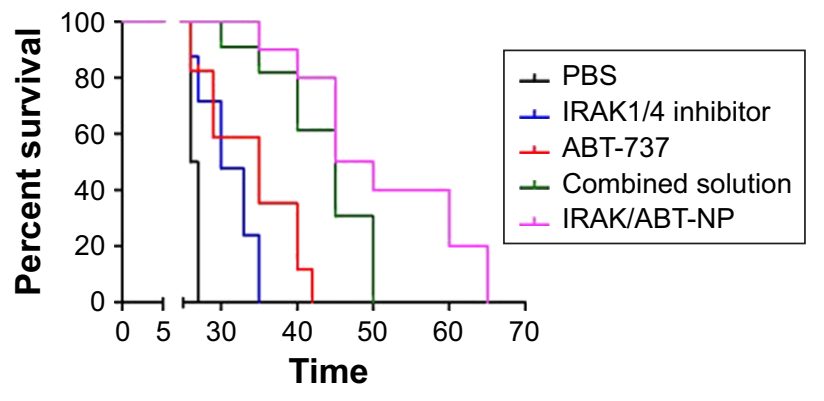

Figure 6 (A) Representative images of peripheral blood smears from T-ALL xenograft mice after treatment with IRAK/ABT-NP, IRAKI/4 inhibitor alone, ABT-737 alone and combined solution. (B) Changes of WBC in T-ALL xenograft mice after treatment with drugs in different formulations. (C) Survival curves in each treated group of mice. Abbreviations: T-ALL, T cell acute lymphoblastic leukemia; IRAK/ABT-NP, IRAKI/4 inhibitor and ABT-737 co-encapsulated into polyethylene glycol modified poly (lacticco-glycolic acid) nanoparticles; WBC, white blood cells. 
rate of IRAK1/4 inhibitor was faster than that of ABT-737, and this was likely due to the smaller molecular weight of the IRAK1/4 inhibitor than ABT-737. Therefore, IRAK1/4 inhibitor could more easily diffuse out from particles. Compared with the combined solution, IRAK/ABT-NP exhibited greater cytotoxicity and induced more apoptosis in the T-ALL cell line. These effects could be explained by IRAK/ABT-NP which had a higher uptake and sustained release in tumor cells.

\section{Conclusion}

In this study, formulation of IRAK/ABT-NP was optimized to achieve high drug loading using BBD-RSM. The optimal IRAK/ABT-NP have a spherical shape and uniform size distribution. In vitro release profiles displayed sustained release of both drugs from the nanoparticles, and a faster release rate of IRAK1/4 inhibitor than ABT-737. IRAK/ABT-NP presented greater killing ability of Jurkat cells than the combined solution. IRAK/ABT-NP also showed better efficacy than the combined solution in treatment of T-ALL mouse model. All these data support our hypothesis that IRAK/ABT-NP may be a promising candidate for therapy of T-ALL.

\section{Acknowledgment}

The authors would like to thank Huazhong University of Science and Technology for providing a supporting research fund.

\section{Disclosure}

The authors report no conflicts of interest in this work.

\section{References}

1. Chiaretti S, Foa R. T-cell acute lymphoblastic leukemia. Haematologica. 2009;94(2):160-162.

2. Dores GM, Devesa SS, Curtis RE, Linet MS, Morton LM. Acute leukemia incidence and patient survival among children and adults in the United States, 2001-2007. Blood. 2012;119(1):34-43.

3. Pui CH. T cell acute lymphoblastic leukemia: NOTCHing the way toward a better treatment outcome. Cancer Cell. 2009;15(2):85-87.

4. Janne PA, Gray N, Settleman J. Factors underlying sensitivity of cancers to small-molecule kinase inhibitors. Nat Rev Drug Discov. 2009;8(9): 709-723.

5. Li Z, Younger K, Gartenhaus R, et al. Inhibition of IRAK1/4 sensitizes $\mathrm{T}$ cell acute lymphoblastic leukemia to chemotherapies. $J$ Clin Invest. 2015;125(3):1081-1097.

6. Cheng H, Addona T, Keshishian H, et al. Regulation of IRAK-4 kinase activity via autophosphorylation within its activation loop. Biochem Biophys Res Commun. 2007;352(3):609-616.

7. Kollewe C, Mackensen AC, Neumann D, et al. Sequential autophosphorylation steps in the interleukin-1 receptor-associated kinase-1 regulate its availability as an adapter in interleukin-1 signaling. $J$ Biol Chem. 2004;279(7):5227-5236.

8. Gelman AE, Zhang J, Choi Y, Turka LA. Toll-like receptor ligands directly promote activated CD4+ T cell survival. J Immunol. 2004;172(10): 6065-6073.
9. Zheng L, Asprodites N, Keene AH, Rodriguez P, Brown KD, Davila E. TLR9 engagement on CD4 T lymphocytes represses gamma-radiationinduced apoptosis through activation of checkpoint kinase response elements. Blood. 2008;111(5):2704-2713.

10. Akira S, Hemmi H. Recognition of pathogen-associated molecular patterns by TLR family. Immunol Lett. 2003;85(2):85-95.

11. Konopleva M, Milella M, Ruvolo P, et al. MEK inhibition enhances ABT-737-induced leukemia cell apoptosis via prevention of ERKactivated MCL-1 induction and modulation of MCL-1/BIM complex. Leukemia. 2012;26(4):778-787.

12. Del Gaizo Moore V, Schlis KD, Sallan SE, Armstrong SA, Letai A. BCL-2 dependence and ABT-737 sensitivity in acute lymphoblastic leukemia. Blood. 2008;111(4):2300-2309.

13. Matsumura Y, Maeda H. A new concept for macromolecular therapeutics in cancer chemotherapy: mechanism of tumoritropic accumulation of proteins and the antitumor agent smancs. Cancer Res. 1986; 46(12 Pt 1):6387-6392.

14. Peer D, Karp JM, Hong S, Farokhzad OC, Margalit R, Langer R. Nanocarriers as an emerging platform for cancer therapy. Nat Nanotechnol. 2007;2(12):751-760.

15. Davis ME, Chen ZG, Shin DM. Nanoparticle therapeutics: an emerging treatment modality for cancer. Nat Rev Drug Discov. 2008;7(9): 771-782.

16. Hu CM, Aryal S, Zhang L. Nanoparticle-assisted combination therapies for effective cancer treatment. Ther Deliv. 2010;1(2):323-334.

17. Livney YD, Assaraf YG. Rationally designed nanovehicles to overcome cancer chemoresistance. Adv Drug Deliv Rev. 2013;65(13-14): $1716-1730$.

18. Sahoo SK, Ma W, Labhasetwar V. Efficacy of transferrin-conjugated paclitaxel-loaded nanoparticles in a murine model of prostate cancer. Int J Cancer. 2004;112(2):335-340.

19. Ye WL, Zhao YP, Li HQ, et al. Doxorubicin-poly (ethylene glycol)alendronate self-assembled micelles for targeted therapy of bone metastatic cancer. Sci Rep. 2015;5:14614.

20. Palamakula A, Nutan MT, Khan MA. Response surface methodology for optimization and characterization of limonene-based coenzyme Q10 self-nanoemulsified capsule dosage form. AAPS PharmSciTech. 2004;5(4):e66.

21. Shaikh J, Ankola DD, Beniwal V, Singh D, Kumar MN. Nanoparticle encapsulation improves oral bioavailability of curcumin by at least 9-fold when compared to curcumin administered with piperine as absorption enhancer. Eur J Pharm Sci. 2009;37(3-4):223-230.

22. Krzek J, Kaleta J, Hubicka U, Niedzwiedz A. Reversed-phase highperformance liquid chromatography determination of selected phenolic acids in propolis concentrates in terms of standardization for drug manufacturing purposes. J AOAC Int. 2006;89(2):352-358.

23. Acharya S, Dilnawaz F, Sahoo SK. Targeted epidermal growth factor receptor nanoparticle bioconjugates for breast cancer therapy. Biomaterials. 2009;30(29):5737-5750.

24. Misra R, Sahoo SK. Intracellular trafficking of nuclear localization signal conjugated nanoparticles for cancer therapy. Eur J Pharm Sci. 2010; 39(1-3):152-163.

25. Dilnawaz F, Singh A, Mohanty C, Sahoo SK. Dual drug loaded superparamagnetic iron oxide nanoparticles for targeted cancer therapy. Biomaterials. 2010;31(13):3694-3706.

26. Kabara E, Coussens PM. Infection of primary bovine macrophages with Mycobacterium avium subspecies paratuberculosis suppresses host cell apoptosis. Front Microbiol. 2012;20(3):215.

27. Liu K, Fang R, Li H, et al. Efficient derivation of embryonic stem cells from NOD-scid I12rg (-/-) mice. Protein Cell. 2015;6(12): 916-918.

28. Jacoby E, Chien CD, Fry TJ. Murine models of acute leukemia: important tools in current pediatric leukemia research. Front Oncol. 2014;4:95.

29. Yang T, Yao S, Zhang X, Guo Y. Andrographolide inhibits growth of human T-cell acute lymphoblastic leukemia Jurkat cells by downregulation of PI3K/AKT and upregulation of p38 MAPK pathways. Drug Des Devel Ther. 2016;10:1389-1397. 
30. Kita K, Dittrich C. Drug delivery vehicles with improved encapsulation efficiency: taking advantage of specific drug-carrier interactions. Expert Opin Drug Deliv. 2011;8(3):329-342.

31. Jaafar-Maalej C, Diab R, Andrieu V, Elaissari A, Fessi H. Ethanol injection method for hydrophilic and lipophilic drug-loaded liposome preparation. J Liposome Res. 2010;20(3):228-243.

32. Prakash P, Kulkarni PP, Chauhan AK. Thrombospondin 1 requires von Willebrand factor to modulate arterial thrombosis in mice. Blood. 2015;125(2):399-406.

33. Kemp JA, Shim MS, Heo CY, Kwon YJ. “Combo” nanomedicine: Co-delivery of multi-modal therapeutics for efficient, targeted, and safe cancer therapy. Adv Drug Deliv Rev. 2016;98:3-18.
34. Cao L, Pan D, Li D, et al. Relation between anti-atherosclerotic effects of IRAK4 and modulation of vascular smooth muscle cell phenotype in diabetic rats. Am J Transl Res. 2016;8(2):899-910.

35. Baranski Z, de Jong Y, Ilkova T, et al. Pharmacological inhibition of Bcl-xL sensitizes osteosarcoma to doxorubicin. Oncotarget. 2015; 6(34):36113-36125.

36. Nii T, Ishii F. Encapsulation efficiency of water-soluble and insoluble drugs in liposomes prepared by the microencapsulation vesicle method. Int J Pharm. 2005;298(1):198-205.

\section{Publish your work in this journal}

The International Journal of Nanomedicine is an international, peerreviewed journal focusing on the application of nanotechnology in diagnostics, therapeutics, and drug delivery systems throughout the biomedical field. This journal is indexed on PubMed Central, MedLine, CAS, SciSearch ${ }^{\circledR}$, Current Contents ${ }^{\circledR} /$ Clinical Medicine,
Journal Citation Reports/Science Edition, EMBase, Scopus and the Elsevier Bibliographic databases. The manuscript management system is completely online and includes a very quick and fair peer-review system, which is all easy to use. Visit http://www.dovepress.com/ testimonials.php to read real quotes from published authors. 